ever-widening degree, every aspect of the reactive event ${ }^{5-7}$.

Molecular beams have a central role in much of this work, and are used to bring together highly directional streams of reactants that intersect in a well-defined region at a specified crossing angle ${ }^{5-7}$. Single reactive collisions or photoexcitation events can then occur, forming products that leave the collision at relative angles and velocities, and with internal state distributions that directly reflect the reactive collision or photoexcitation that spawned them. Techniques to achieve this include supersonically expanding a gas to cool the reactant molecules' vibrations to their lowest quantum-mechanically allowed levels, and velocity selection, which serves the purpose of defining the initial speed of approach.

Whereas early molecular-beam studies focused on simple reactions involving small molecules, in recent years molecules of increasing complexity have been the subject of such approaches ${ }^{7}$. A fascinating aspect of this push towards greater complexity is the increasingly pervasive presence of conformational isomers of the molecules of interest. These isomers differ from one another in their molecular shapes, with their interconversion involving internal rotation of molecular subgroups about one or more their bonds (Fig. 1). This seemingly innocuous reorientation of subgroups in the molecule has direct and sometimes profound consequences for the way the molecule interacts, either reactively or non-reactively, with other molecules. It is thus an exciting prospect to study, under isolated-molecule or single-collision conditions, the spectroscopic signatures, interconversion, intermolecular binding and chemical reactivity of individual conformational isomers.

The challenge is to select out of the mixture the conformer of interest. This is no small feat because all conformers have the same mass, same molecular formula and same chemical connectivity. The method described by Küpper, Stapelfeldt and co-workers ${ }^{4}$ uses an inhomogeneous electric field to steer neutral molecules, using their unequal internal charge distribution, perpendicular to the direction of motion of the molecular beam (Fig. 1). The technique of Stark deflection has a long history ${ }^{8}$, but its application in this recent research ${ }^{4}$ to selectively steer one stereoisomer in a mixture to the interaction region is a tour de force of modern molecular-beam wizardry.

The researchers used 3-aminophenol in their demonstration experiment, whose cis- and trans-isomers (Fig. 1) possess very different dipole moments (2.33 debye and 0.77 debye, respectively) that lead to differing degrees of deflection, causing a separation in the beam trajectories for the two isomers. As the two isomers have unique ultraviolet spectra, the make-up of the beam can be probed using a laser-based two-photon ionization scheme that uses each isomer's excited electronic state as an intermediate state, detecting the isomerspecific ions produced by the laser in a mass spectrometer. They achieve an impressive degree of control over the isomeric make-up of the beam arriving at the laser interaction region, varying from nearly pure cis- to nearly pure trans-isomers, or anywhere in between. This method is complementary to a dynamic focusing alternative demonstrated recently by the same group involving high-voltage a.c. fields ${ }^{9}$.

Having achieved this milestone of isomeric control, prospects are bright for a number of ground-breaking studies that can build on this work. As the methods for cooling and steering improve, even the alignment and orientation of the individual isomers become non-uniform, so molecules could be directed to approach one another with dipole moments aligned, anti-aligned or perpendicular to one another. Among the more exotic possibilities would be to use these spatially separated and aligned, isomerically pure samples for X-ray diffraction studies of large gas-phase molecules.

It is still unclear just how far down this road towards isomer-specific chemical dynamics one can go, as the isomeric makeup of the sample becomes more complex and diverse. What is clear is that - where molecular beams are involved - travelling this road will probably involve a bit of molecular-scale steering.

Timothy S. Zwier is in the Department of Chemistry, Purdue University, West Lafayette, Indiana 47907-2084, USA.

e-mail:zwier@purdue.edu

\section{References}

1. Simons, J. P. et al. Int. Rev. Phys. Chem. 24, 489-531 (2005).

2. Choi, M. Y. et al. Int. Rev. Phys. Chem. 25, 15-75 (2006).

3. Zwier, T. S. J. Phys. Chem. A 110, 4133-4150 (2006).

4. Filsinger, F. et al. Angew. Chem. Int. Ed. 48, 6900-6902 (2009).

5. Lee, Y. T. Angew. Chem. Int. Ed. 26, 939-951 (1987).

6. Herschbach, D. R. Ann. Rev. Phys. Chem. 51, 1-39 (2000)

7. Gu, X. B. \& Kaiser, R. I. Acc. Chem. Res. 42, 290-302 (2009).

8. Klemperer, W., Lehmann, K. K., Watson, J. K. G. \& Wofsy, S. C. J. Phys. Chem. 97, 2413-2416 (1993).

9. Filsinger, F., Erlekam, U., von Helden, G., Kupper, J. \& Meijer, G. Phys. Rev. Lett. 100, 133003 (2008)

\title{
MICROSTRUCTURE GROWTH
}

\section{Promoting precipitation}

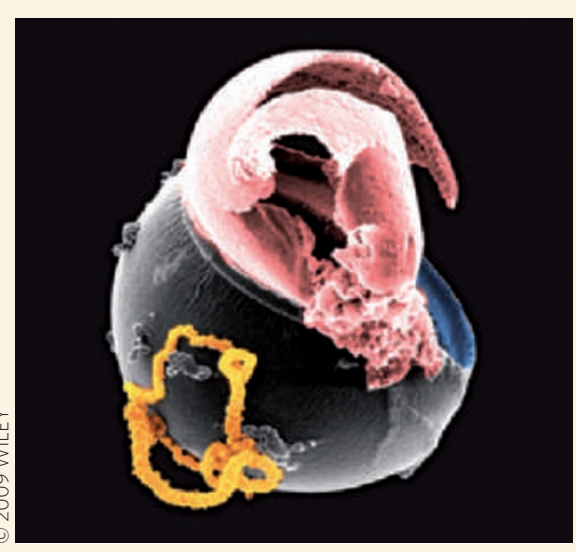

Many chemists are aware of the 'chemical garden' demonstration, in which structures that look similar to plants grow from solution. The growths are millimetrescale hollow tubes that form through a precipitation reaction, which occurs on the addition of crystals of soluble metal salts to solutions of anions such as silicates, borates or carbonates. Now, Oliver Steinbock and colleagues at Florida State University have modified this approach to gain greater control and understanding of the tubular growths, which could eventually lead to applications in microfluidic devices (Angew. Chem. Int. Ed. 48, 8752-8756; 2009).
They use agarose beads that are loaded with copper sulfate solution as reactant vessels that seed the precipitation growth of micrometre-scale tubes (orange in the picture). The beads are exposed to a sodium silicate solution and microtubes grow out from a bubble on their surface. The bubble is attached to the end of the growing microtube and either the bubble or the bead moves as the tube grows. Nodular objects (shown in pink) that have a completely different morphology to the tubes are also seen to grow from the beads.

GAVIN ARMSTRONG 